\title{
A Note Concerning the Properties of a Certain Class of Test Matrices
}

\section{By Harold Willis Milnes}

1. Introduction. The number of matrices for which the properties may be expressed analytically is surprisingly small. The list is limited essentially to diagonal matrices, triangular forms, circulants, Hilbert matrices, and a few others. In this paper we describe one more class of matrices for which such properties as the characteristic equation, eigenvectors, associated determinant and inverse matrix can be found in a comparatively simple way. These matrices are quite valuable in providing test matrices for evaluating the accuracy and efficiency of computational procedures. They are especially useful in this regard since they may be chosen of arbitrary order, and by appropriately selecting their elements they may be made as nearly singular as may be desired. It is thus possible, for instance, to rate various numerical methods of matrix inversion by comparing computed inverses with the known exact expressions for the inverse.

2. The Matrix and Its Inverse. We shall denote the matrix of order $n$, with which we are concerned as

$$
K_{n} \equiv K_{n}\left(a_{1}, a_{2}, \cdots, a_{n-1}\right)=\left(\alpha_{i j}\right), \quad(i, j=1,2, \cdots, n),
$$

where $a_{1}, a_{2}, \cdots, a_{n-1}$ are constants and

$$
\begin{aligned}
\alpha_{i j} & =1, & & j \geqq i, \\
& =a_{j}, & & j<i .
\end{aligned}
$$

Thus

$$
\begin{array}{cl}
K_{1}=[1], & \iota_{2}=\left[\begin{array}{ll}
1 & 1 \\
a_{1} & 1
\end{array}\right], K_{3}=\left[\begin{array}{lll}
1 & 1 & 1 \\
a_{1} & 1 & 1 \\
a_{1} & a_{2} & 1
\end{array}\right], \\
K_{4}=\left[\begin{array}{lllll}
1 & 1 & 1 & 1 \\
a_{1} & 1 & 1 & 1 \\
a_{1} & a_{2} & 1 & 1 \\
a_{1} & a_{2} & a_{3} & 1
\end{array}\right], & K_{5}=\left[\begin{array}{lllll}
1 & 1 & 1 & 1 & 1 \\
a_{1} & 1 & 1 & 1 & 1 \\
a_{1} & a_{2} & 1 & 1 & 1 \\
a_{1} & a_{2} & a_{3} & 1 & 1 \\
a_{1} & a_{2} & a_{3} & a_{4} & 1
\end{array}\right] .
\end{array}
$$

The inverse of these matrices may be written down immediately according to a remarkably simple formula as

$$
K_{n}^{-1}=\left(\beta_{i j}\right)
$$

where 


$$
\begin{aligned}
\beta_{i j} & =0, \quad j>i+1, i<n-1(n=2,3, \cdots) \\
& =0, \quad j<i, i \neq n, \\
& =1 /\left(1-a_{i}\right), \quad j=i, i \neq n, \\
& =-1 /\left(1-a_{i}\right), \quad j=i+1, i \neq n, \\
& =\left(a_{j-1}, a_{j}\right) /\left(1-a_{j}\right)\left(1-a_{j-1}, \quad j \neq 1, n, i=n,\right. \\
& =-a_{1} /\left(1-a_{1}\right), \quad j=1, i=n, \\
& =1 /\left(1-a_{n-1}\right), \quad j=n, i=n, \\
\beta_{i j} & =1, \quad(n=1) .
\end{aligned}
$$

Thus

$$
\begin{aligned}
& K_{1}^{-1}=[1], \quad K_{2}^{-1}=\left[\begin{array}{cc}
\frac{1}{\left(1-a_{1}\right)} & \frac{-1}{\left(1-a_{1}\right)} \\
\frac{-a_{1}}{\left(1-a_{1}\right)} & \frac{1}{\left(1-a_{1}\right)}
\end{array}\right] \text {, } \\
& K_{3}^{-1}=\left[\begin{array}{ccc}
\frac{1}{\left(1-a_{1}\right)} & \frac{-1}{\left(1-a_{1}\right)} & 0 \\
0 & \frac{1}{\left(1-a_{2}\right)} & \frac{-1}{\left(1-a_{2}\right)} \\
\frac{-a_{1}}{\left(1-a_{1}\right)} & \frac{\left(a_{1}-a_{2}\right)}{\left(1-a_{1}\right)\left(1-a_{2}\right)} & \frac{1}{\left(1-a_{2}\right)}
\end{array}\right], \\
& K_{4}^{-1}=\left[\begin{array}{cccc}
\frac{1}{\left(1-a_{1}\right)} & \frac{-1}{\left(1-a_{1}\right)} & 0 & 0 \\
0 & \frac{1}{\left(1-a_{2}\right)} & \frac{-1}{\left(1-a_{2}\right)} & 0 \\
0 & 0 & \frac{1}{\left(1-a_{3}\right)} & \frac{-1}{\left(1-a_{3}\right)} \\
\frac{-a_{1}}{\left(1-a_{1}\right)} & \frac{\left(a_{1}-a_{2}\right)}{\left(1-a_{1}\right)\left(1-a_{2}\right)} & \frac{\left(a_{2}-a_{3}\right)}{\left(1-a_{2}\right)\left(1-a_{3}\right)} & \frac{1}{\left(1-a_{3}\right)}
\end{array}\right] \text {, } \\
& K_{5}^{-1}=\left[\begin{array}{ccccc}
\frac{1}{\left(1-a_{1}\right)} & \frac{-1}{\left(1-a_{1}\right)} & 0 & 0 & 0 \\
0 & \frac{1}{\left(1-a_{2}\right)} & \frac{-1}{\left(1-a_{2}\right)} & 0 & 0 \\
0 & 0 & \frac{1}{\left(1-a_{3}\right)} & \frac{-1}{\left(1-a_{3}\right)} & 0 \\
0 & 0 & \frac{1}{\left(1-a_{4}\right)} & \frac{-1}{\left(1-a_{4}\right)} \\
\frac{-a_{1}}{\left(1-a_{1}\right)} \frac{\left(a_{1}-a_{2}\right)}{\left(1-a_{1}\right)\left(1-a_{2}\right)} \frac{\left(a_{2}-a_{3}\right)}{\left(1-a_{2}\right)\left(1-a_{3}\right)} \frac{\left(a_{3}-a_{4}\right)}{\left(1-a_{3}\right)\left(1-a_{4}\right)} \frac{1}{\left(1-a_{4}\right)}
\end{array}\right] \text {. }
\end{aligned}
$$


The proof may be made by direct matrix multiplication, for if

$$
K_{n} K_{n}^{-1} \equiv\left(\gamma_{i j}\right)
$$

then

$$
\begin{aligned}
\gamma_{11} & =\frac{1}{\left(1-a_{1}\right)}-\frac{a_{1}}{\left(1-a_{1}\right)}=1, \\
\gamma_{i 1} & =\frac{a_{1}}{\left(1-a_{1}\right)}-\frac{a_{1}}{\left(1-a_{1}\right)}=0 \quad(i=2, \cdots n), \\
\gamma_{i i} & =\frac{-a_{i-1}}{\left(1-a_{i-1}\right)}+\frac{1}{\left(1-a_{i}\right)}+\frac{a_{i-1}-a_{i}}{\left(1-a_{i-1}\right)\left(1-a_{i}\right)}=1 \quad(i=2, \cdots, n-1), \\
(2.8) \quad \gamma_{n n} & =\frac{-a_{n-1}}{\left(1-a_{n-1}\right)}+\frac{1}{\left(1-a_{n-1}\right)}=1, \\
\gamma_{i j} & =\frac{-a_{j-1}}{\left(1-a_{j-1}\right)}+\frac{a_{j}}{\left(1-a_{j}\right)}+\frac{\left(a_{j-1}-a_{j}\right)}{\left(1-a_{j-1}\right)\left(1-a_{j}\right)}=0 \quad(j<i, j \neq 1), \\
\gamma_{i j} & =-\frac{1}{\left(1-a_{j-1}\right)}+\frac{1}{\left(1-a_{j}\right)}+\frac{\left(a_{j-1}-a_{j}\right)}{\left(1-a_{j-1}\right)\left(1-a_{j}\right)}=0 \quad(j>i, j \neq n), \\
a_{i n} & =-\frac{1}{\left(1-a_{n-1}\right)}+\frac{1}{\left(1-a_{n-1}\right)}=0 \quad(i \neq n) .
\end{aligned}
$$

3. The Determinant. The determinant of $K_{n}$ is easily evaluated for if we write $\operatorname{det}\left(K_{n}\right)$ as

$$
\operatorname{det}\left(K_{n}\right)=\left[\begin{array}{lllllll}
1 & 1 & 1 & 1 & --- & 1 & 1 \\
a_{1} & 1 & 1 & 1 & --- & 1 & 1 \\
a_{1} & a_{2} & 1 & 1 & --- & 1 & 1 \\
a_{1} & a_{2} & a_{3} & 1 & --- & 1 & 1 \\
- & - & - & - & --- & - & 1 \\
a_{1} & a_{2} & a_{3} & a_{4} & --- & a_{n-1} & 1
\end{array}\right]
$$

then by subtracting $a_{j}$ times the last column from the $j$ th column $(j=1,2, \cdots,(n-1))$, we find

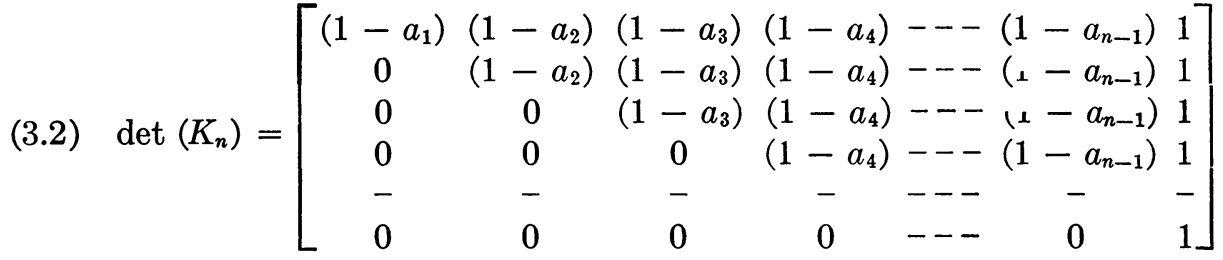

$$
\begin{aligned}
& \equiv\left(1-a_{1}\right)\left(1-a_{2}\right)\left(1-a_{3}\right)\left(1-a_{4}\right)---\left(1-a_{n-1}\right) \text {. }
\end{aligned}
$$

It is clear that $K_{n}$ is singular if and only if one or more of the $a$ 's is equal to unity.

4. Characteristic Vectors and Characteristic Equation. Unless $a_{j}=1$ for some $j$, det $\left(K_{n}\right)=\lambda_{1}, \lambda_{2}, \cdots, \lambda_{n} \neq 0$ where the $\lambda$ 's are characteristic values of $K_{n}$. 
Assuming that $a_{j} \neq 1$, it is possible to determine the characteristic vectors associated with a given characteristic value. We consider such a vector in the form

$$
\mathbf{v}=\left\{1, x_{2}, x_{3}, \cdots, x_{n}\right\}^{T}
$$

and write the equation

$$
K_{n} \mathbf{v}=\lambda \mathbf{v}
$$

as the linear system

$$
\begin{gathered}
1+x_{2}+x_{3}+\cdots+x_{n}=\lambda, \\
a_{1}+x_{2}+x_{3}+\cdots+x_{n}=\lambda x_{2}, \\
a_{1}+a_{2} x_{2}+x_{3}+\cdots+x_{n}=\lambda x_{3}, \\
-\quad-\quad-\cdots-- \\
a_{1}+a_{2} x_{2}+a_{3} x_{3}+\cdots+x_{n}=\lambda x_{n} .
\end{gathered}
$$

Subtracting the first equation from the second and solving for $x_{2}$ gives

$$
x_{2}=\left(\lambda-1+a_{1}\right) / \lambda .
$$

Subtracting the second equation from the third and solving for $x_{3}$ gives

$$
x_{3}=\left(\lambda-1+a_{2}\right) x_{2} / \lambda=\left(\lambda-1+a_{1}\right)\left(\lambda-1+a_{2}\right) / \lambda^{2} .
$$

Proceeding similarly by subtracting the $(j-1)$ th equation from the $j$ th and solving for $x_{j}(j=2,3, \cdots, n)$, we find

$$
x_{j}=\left(\lambda-1+a_{j}\right) x_{j-1} / \lambda=\lambda^{-(j-1)} \prod_{k=1}^{j-1}\left(\lambda-1+a_{k}\right)
$$

and the characteristic vector is obtained.

We observe that the above process is always uniquely determined and yields one characteristic vector for each distinct nonzero value of $\lambda$. Thus, if $\lambda \neq 0$ is a repeated characteristic value of multiplicity $\alpha$, there is but one characteristic vector associated with the value of $\lambda$.

Substituting the values for $x_{2}, x_{3}, \cdots, x_{n}$ into the first equation of (4.3), we obtain the following relation

$$
\begin{aligned}
& \lambda=1+\frac{\left(\lambda-1+a_{1}\right)}{\lambda}\left(1+\frac{\left(\lambda-1+a_{2}\right)}{\lambda}\left(1+\frac{\left(\lambda-1+a_{3}\right)}{\lambda}(\ldots\right.\right. \\
& \left.\left.\left.\left(1+\frac{\left(\lambda-1+a_{n-1}\right)}{\lambda}\right) \ldots\right)\right)\right) \\
& =\frac{1}{\lambda^{n-1}}\left[\lambda^{n-1}+\lambda^{n-2}\left(\lambda-1+a_{1}\right)+\lambda^{n-3} \prod_{j=1}^{2}\left(\lambda-1+a_{j}\right)\right. \\
& +\lambda^{n-4} \prod_{j=1}^{3}\left(\lambda-1+a_{j}\right)+\cdots+\lambda \prod_{j=1}^{n-2}\left(\lambda-1+a_{j}\right) \\
& \left.+\prod_{j=1}^{n-1}\left(\lambda-1+a_{j}\right)\right] \text {. }
\end{aligned}
$$


We note that $\prod_{j=1}^{k}\left(\lambda-1+a_{j}\right)$ is a polynomial in $\lambda$ of degree $k$ with roots $\left(1-a_{1}\right),\left(1-a_{2}\right), \cdots,\left(1-a_{k}\right)$ and we write it as

$$
\prod_{j=1}^{k}\left(\lambda-1+a_{j}\right)=\lambda^{k}-S_{1, k} \lambda^{k-1}+S_{2, k} \lambda^{k-2}-\cdots+(-1)^{k} S_{k, k}
$$

Here $S_{j, k}$ stands for

$$
S_{j k}=\sum\left(1-a_{\alpha_{1}}\right)\left(1-a_{\alpha_{2}}\right) \cdots\left(1-a_{\alpha_{j}}\right),
$$

where the sum extends over all possible products of the $k$ terms $\left(1-a_{1}\right),\left(1-a_{2}\right)$, $\cdots,\left(1-a_{k}\right)$ taken in combinations $j$ at a time. Substituting (4.8) in (4.7) and multiplying (4.7) by $\lambda^{n-1} \neq 0$, the characteristic equation of $K_{n}$ is seen to be

$$
\begin{aligned}
\lambda^{n}-n \lambda^{n-1} & +\left(S_{1,1}+S_{1,2}+\cdots+S_{1, n-1}\right) \lambda^{n-2}, \\
& -\left(S_{2,2}+S_{2,3}+\cdots+S_{2, n-1}\right) \lambda^{n-3}, \\
& +\left(S_{3,3}+S_{3,4}+\cdots+S_{3, n-1}\right) \lambda^{n-4}, \\
& ------------- \\
& (-1)^{n} S_{n-1, n-1}=0 .
\end{aligned}
$$

If $P_{j}=P_{j}(\lambda)$ denotes the polynomial of degree $j$ which has the roots $\left(1-a_{1}\right)$, $\left(1-a_{2}\right), \cdots,\left(1-a_{j}\right)$ then the characteristic equation can be otherwise expressed as

$$
\lambda^{n}-\lambda^{n-1}-\lambda^{n-2} P_{1}(\lambda)-\lambda^{n-3} P_{2}(\lambda) \cdots-P_{n-1}(\lambda)=0 .
$$

When any of the characteristic roots is zero, then we have seen that $a_{j}=1$ for certain values of $j$. This implies that the corresponding $j$ th column of $K_{n}$ is identical with the last column. To compute the characteristic equation and vectors we may simply cross out the offending rows and columns and proceed as before in the reduced space.

The resulting characteristic equation is afterwards multiplied by a suitable factor of $\lambda$ and the characteristic vectors expanded to a full complement, by adjoining a basis set for the null space of $K_{n}$. An illustrative example will suffice to illustrate how this is done.

Example.

$$
K_{4}=\left[\begin{array}{llll}
1 & 1 & 1 & 1 \\
a & 1 & 1 & 1 \\
a & 1 & 1 & 1 \\
a & 1 & 1 & 1
\end{array}\right]
$$

We consider $K_{2}$ obtained by striking out the 2 nd and 3 rd rows and columns

$$
K_{2}=\left[\begin{array}{ll}
1 & 1 \\
a & 1
\end{array}\right]
$$

This has the characteristic equation

$$
(\lambda-1)^{2}-a=0
$$

with roots $1+\sqrt{ } a, 1-\sqrt{ } a$, and the characteristic vectors of $K_{2}$ are 


$$
\left[\begin{array}{l}
1 \\
\sqrt{ } a
\end{array}\right], \quad\left[\begin{array}{l}
1 \\
-\sqrt{ } a
\end{array}\right] .
$$

The characteristic equation of $K_{4}$ is

$$
\lambda^{2}\left(\lambda^{2}-2 \lambda+1-a\right)=0
$$

and the characteristic vectors are

$$
\left[\begin{array}{l}
1 \\
\sqrt{ } a \\
0 \\
0
\end{array}\right], \quad\left[\begin{array}{l}
1 \\
-\sqrt{ } a \\
0 \\
0
\end{array}\right], \quad\left[\begin{array}{l}
0 \\
0 \\
1 \\
0
\end{array}\right], \quad\left[\begin{array}{l}
0 \\
0 \\
0 \\
1
\end{array}\right] .
$$

We observe in this example that if $a=0$, then $\lambda=1$ has multiplicity 2 , and one of the characteristic vectors is dependent.

5. A Test Matrix. As commented in the introduction, the matrices $K_{n}$ are useful test matrices for evaluating matrix computational procedures. Such test matrices may be constructed in a variety of ways by giving numerical values to $a_{1}, a_{2}, \cdots, a_{n-1}$. We have used the particular choice (5.1), since its inverse is especially simple and since its determinant has the value $10^{-n(n+1) / 2}$ the matrix approaches singularity rapidly for small values of $n$, while the elements of both the direct and inverse matrices remain within reasonable bounds. This test matrix is denoted by $T_{n}$ and is given with its inverse as

$$
\begin{aligned}
T_{n} & =\left[\begin{array}{llllll}
1 & 1 & 1 & 1 & \ldots & 1 \\
.9 & 1 & 1 & 1 & \ldots & 1 \\
.9 & .99 & 1 & 1 & \ldots & 1 \\
.9 & .99 & .999 & 1 & \ldots & 1 \\
- & - & - & - & - & - \\
.9 & .99 & .999 & .9999 & \ldots & 1
\end{array}\right], \\
T_{n}{ }^{-1} & =\left[\begin{array}{rrrrrr}
10 & -10 & 0 & 0 & \ldots & 0 \\
0 & 100 & -100 & 0 & \ldots & 0 \\
0 & 0 & 1000 & -1000 & \ldots & 0 \\
0 & 0 & 0 & 10000 & \ldots & 0 \\
- & - & - & - & - & - \\
-9 & -90 & -900 & -9000 & \ldots & 10^{n-1}
\end{array}\right],
\end{aligned}
$$

Texas Technological College

Lubbock, Texas 79407 